\title{
Design and Evaluation of a 10-mA DC Current Reference Standard
}

\author{
Gunnar Fernqvist, Member, IEEE, Gregory Hudson, John Pickering, Member, IEEE, and Francis Power
}

\begin{abstract}
A new dc current reference standard has been developed for high-current power converter calibration in the large hadron collider (LHC) project at the European Organization for Nuclear Research (CERN). This standard provides a near ideal 10-mA dc current with long-term drift of one part in $10^{6}$ per year. The paper describes the requirements and the detailed design and evaluation of the unit. Since similar 10-V standards are commercially available, the paper concentrates on the unique current output capability of this device.
\end{abstract}

Index Terms-Current source, dc current calibration, reference standard, zener reference.

\section{INTRODUCTION}

$\mathbf{T}$ HE large hadron collider (LHC) project at the European Organization for Nuclear Research (CERN) [1] requires an unprecedented accuracy, a few parts in $10^{6}$, in the control of the 13-kA current to the superconducting magnets. To achieve this aim, a new calibration infrastructure is being built, based on a $10-\mathrm{mA} \mathrm{dc}$ current reference and transfer standard. It provides a fully floating $10-\mathrm{mA} \mathrm{dc}$ current output with $11 \mathrm{~V}$ compliance and many hours of battery autonomy. It also provides an accurate $10-\mathrm{V}$ output in a manner similar to existing products such as the Fluke 7000 [2] and 732B. No comparable current standards existed previously and, therefore, this difficult additional capability had to be developed.

\section{RATIONALE}

The ideas and principles for the whole calibration system were developed in [3] and only the beginning of the calibration chain is summarized here. It was realized from the outset that there was a need for central fixed standards as well as portable units. The value of $10 \mathrm{~mA}$, based on engineering considerations, seemed suitable for all of the following applications, allowing a single design of dc current reference, referred to as the PBC, to cater for each.

Reference Standard: A number of reference standards (5) will be kept in the CERN standards laboratory under near ideal conditions. Periodic comparisons will be made against primary standards at the Swiss Federal Office for Metrology and Accreditation (METAS) with the help of $10-\mathrm{V}$ voltage standards and $1-\Omega-1-\mathrm{k} \Omega$ resistance standards to ensure traceability. Periodic inter-comparisons provide short-term stability data. Uniquely, the PBC allows current to voltage transfers to

Manuscript received June 17, 2002; revised October 15, 2002.

G. Fernqvist G. Hudson and F. Power are with the European Organization for Nuclear Research (CERN), Geneva, Switzerland.

J. Pickering is with Metron Designs Ltd., Norwick, U.K.

Digital Object Identifier 10.1109/TIM.2003.809915 be made via a $1-\mathrm{k} \Omega$ standard resistor, whilst simultaneously performing a current transfer to another PBC.

Travelling standards: A small number of standards are dedicated to transferring the $10 \mathrm{~mA}$ to the 18 sites located $100 \mathrm{~m}$ underground around the $27-\mathrm{km}$ collider ring.

Local current source: A 10-mA current source is needed in the current calibrator [4], which is a turns ratio device able to multiply the reference current by a factor up to a 1000 with more than 24 bits resolution. The 5-A output current is used for calibrating large dc current transducers in the power converters, which are, in turn, used for the final transfer to $13 \mathrm{kA}$.

\section{Design Reguirements AND Performance}

\section{A. Accuracy/Stability}

The target is to deliver consistent magnet current accuracy of a few parts in $10^{6}$ under all conditions and with many contributing system uncertainties. The primary calibration support chain therefore needs a good margin for confidence and to extend calibration intervals. These considerations demanded performance comparable, in current, with the very best dc voltage references, i.e., short term stability about two parts in $10^{7}$ and long term drift about one part in $10^{6}$ per year.

1) Noise: To evaluate the dc current output noise, pairs of PBCs were connected back-to-back and the difference current measured. As the noise from different units is uncorrelated and the method actually represents the intended transfer method, it gives a good picture of operational performance (see Fig. 1 for typical behavior). The variations from unit to unit were small. The $10-\mathrm{V}$ voltage output noise was also verified in a conventional manner. It was found to be very similar to the current output in relative performance. In fact, the noise in both the current and voltage outputs originates mainly from the Zener voltage reference, with some units exhibiting additional noise generated in the current converter circuits.

2) Time stability: The five prototype units were each connected to a $100-\Omega$ resistor and measured daily with an MIL 8000 against Fluke 732B standards traceable to METAS/Bern. The long term drift can be seen in Fig. 2. When the measurement resistors were replaced by $1 \mathrm{k} \Omega$, the inter-comparison could be made directly with the Fluke 732B 10-V standards through the DataProof scanner and uncertainty reduced. It is estimated that a group of four units can easily provide the $10 \mathrm{~mA}$ with an uncertainty of five parts in $10^{7}$ constantly maintained. The five units were also compared against each other periodically and the results agree very well with the 


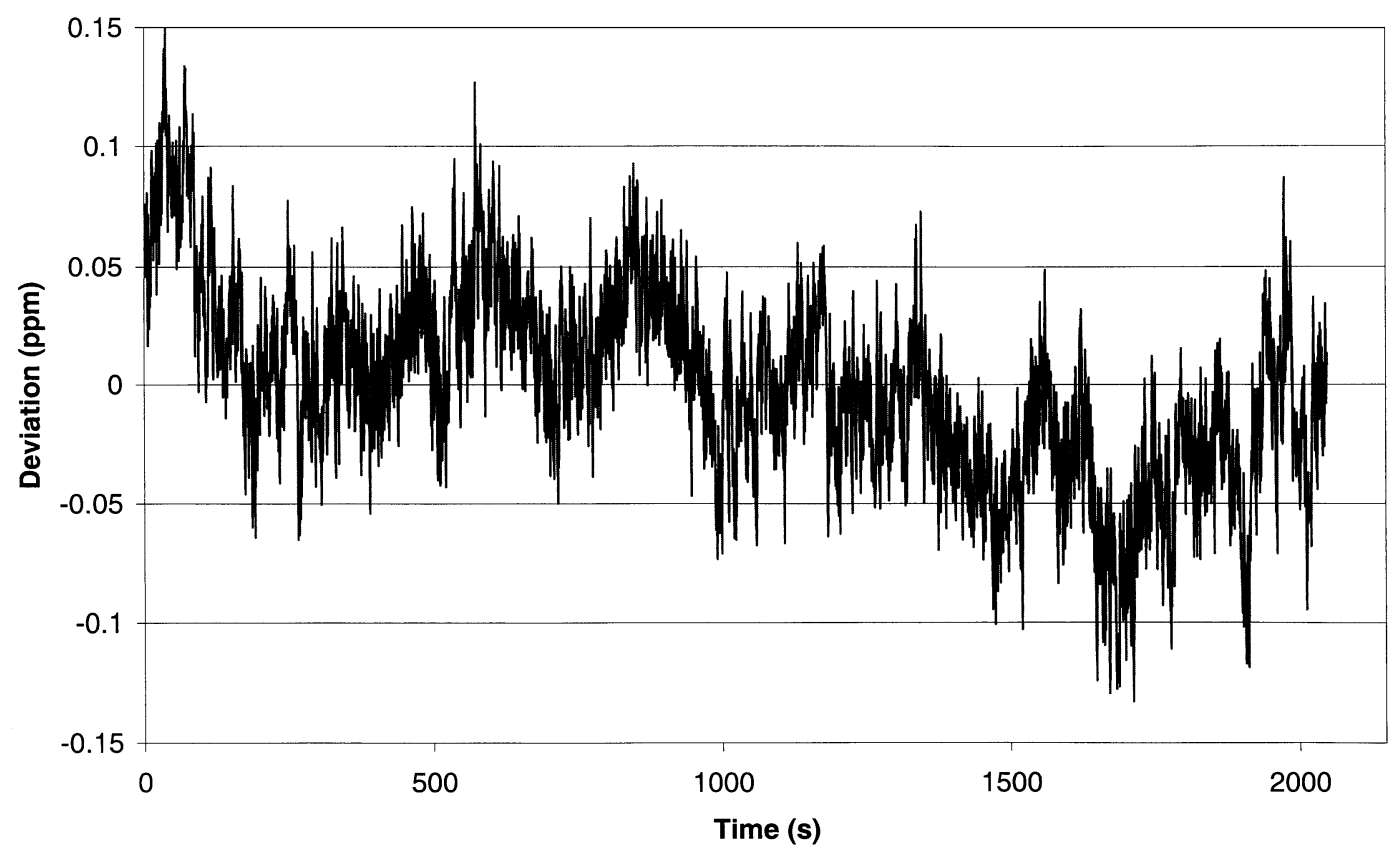

Fig. 1. Medium term noise in the 10-mA output.

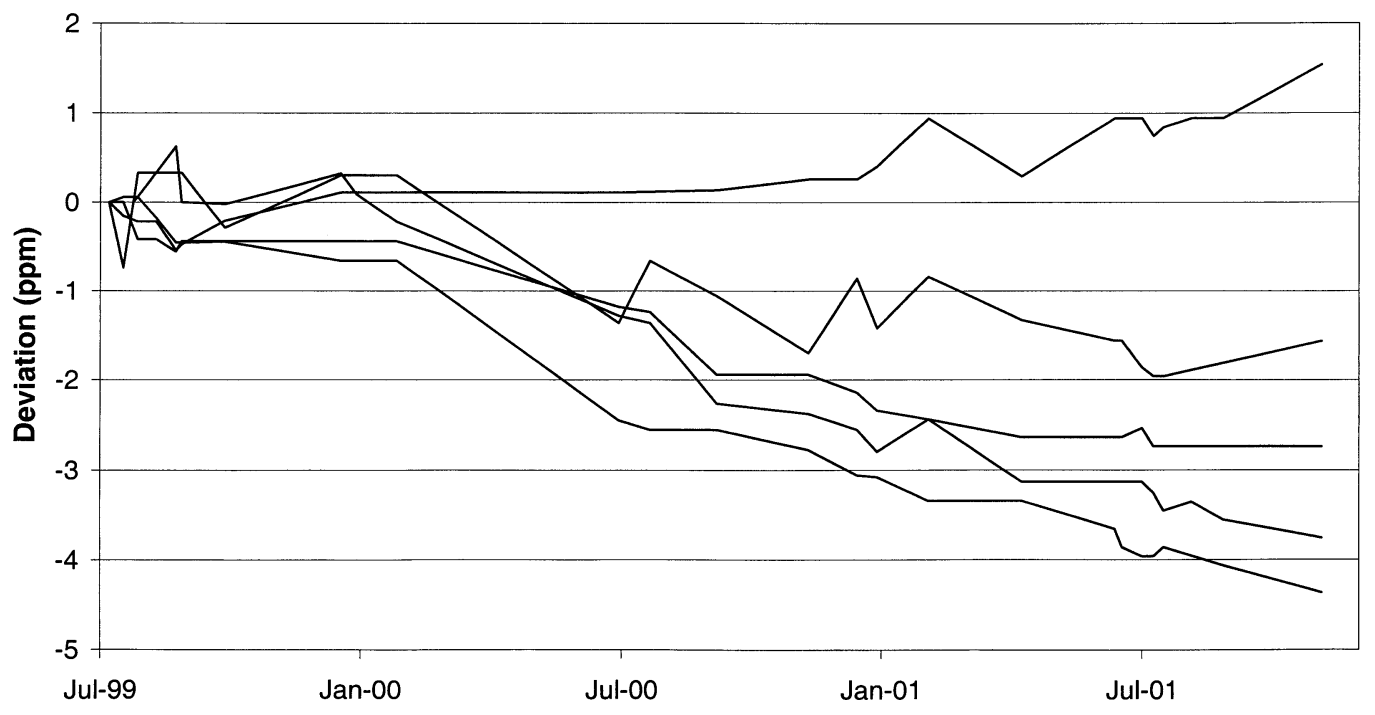

Fig. 2. Long term drift of the $10-\mathrm{mA}$ output.

above method, increasing confidence in the technique. The voltage outputs were also monitored periodically, but only with the intention to discern the origin of drift in the current output. After initial settling, the current output drift generally follows the voltage output.

3) Temperature stability: Temperature coefficient (TC) was measured with the back-to-back technique, one unit at fixed temperature and the other one at a variable temperature. The units were measured at $13,18,23,28$, and $33^{\circ} \mathrm{C}$. The TC of the current output was typically $7 \times 10^{-8} / \mathrm{K}$ (max. $\left.1.4 \times 10^{-7} / \mathrm{K}\right)$ and of the voltage output typically $2 \times 10^{-8} / \mathrm{K}\left(\max .6 \times 10^{-8} / \mathrm{K}\right)$.

\section{B. Compliance}

The PBC output will be used with at least three different types of loads: 1) For calibration against primary standards, it will see resistive loads up to $1 \mathrm{k} \Omega$ while still being able, concurrently, to perform back-to-back calibration of another PBC. 2) At an inter-comparison between two PBCs, it will see $0 \mathrm{~V}$ at a very high impedance. 3) Feeding the current calibrator, it will see a resistive and inductive load of up to $70 \Omega / 50 \mathrm{mH}$.

The first calibrations and long-term drift evaluations were made with a $100-\Omega$ resistor in the output and at the $1-\mathrm{V}$ level to minimize power in the standard resistor. It was realized that if $1-\mathrm{k} \Omega$ standard resistors capable of sustaining $100 \mathrm{~mW}$ continuously could be used, a better configuration would be to leave them permanently connected, even during intercomparison between the reference PBCs and the travelling units. The new configuration improved the overall uncertainty.

The compliance was tested by connecting two PBCs back-to-back with a 1-M $\Omega$ load. A variable resistor was put in series with one PBC to provide a voltage drop. The stability was 


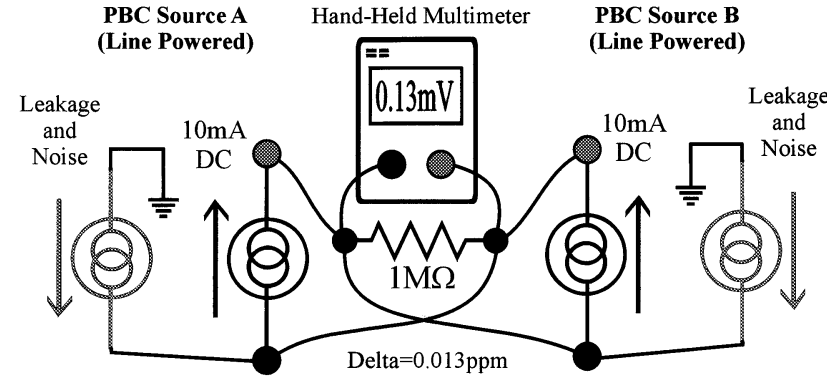

Fig. 3. Back-to-back comparison of PBCs.

easily observed across the 1-M $\Omega$ resistor $(1 \mathrm{mV}$ corresponds to one part in $10^{7}$ ). Over the $0-10 \mathrm{~V}$ range the change was less than one part in $10^{7}$ and saturation started at $10.2 \mathrm{~V}$. This margin was felt too small and the design updated to improve this to greater than $11 \mathrm{~V}$. Second, the compliance dynamic performance was tested, again using back-to-back, with the load on one of the PBCs being the CERN current calibrator. The winding resistance and inductance changes as windings are switched in and out, but no instability or permanent change was observed in the PBC output.

\section{Isolation}

Since calibration transfers between units are performed by reverse connecting them and sensing the current difference to $0.1 \mathrm{nA},\left(1 \mathrm{nA}\right.$ corresponds to one part in $\left.10^{7}\right)$ it is critically important that power supply current does not interfere. This requires very low leakage current back to ground via the external supply. Even the ac leakage current has to be kept very low to ensure that it does not interfere with the current null measurement (see Fig. 3). The measured rms noise in this configuration was $<10 \mathrm{nA}$, mainly $8 \mathrm{kHz}$ from the internal $\mathrm{dc} / \mathrm{dc}$ converter.

\section{Portability}

The autonomy should permit travelling around the LHC during a full working day without interruption i.e., $>10 \mathrm{~h}$ including margin. The autonomy of the initial units decreased with time due to insufficient trickle charging and is now only $6-9 \mathrm{~h}$ The charging circuit was redesigned and the autonomy is now $>16 \mathrm{~h}$. Built-in monitoring provides warning if the battery is low $1 / 2 \mathrm{~h}$ before the unit stops functioning. A warning light indicates if the temperature control is lost, i.e., ambient temperature is outside the specified range or a hardware failure has occurred. Mains power was removed and returned several hours later. The change was less than one part in $10^{7}$. The batteries were allowed to discharge completely and the units were re-powered. An LED indicated that power had previously failed, but after resetting with the front panel "calibration reset," the retrace was much better than five parts in $10^{7}$.

\section{DESIGN DESCRIPTION}

As shown in Fig. 4, the basic "Voltage" part of the design is similar to that of a commercial dc voltage source [2] with a number of modifications to generate the $10-\mathrm{mA}$ reference current and meet the above requirements. The gain step to $10 \mathrm{~V}$ from the well-known and predictable zener reference

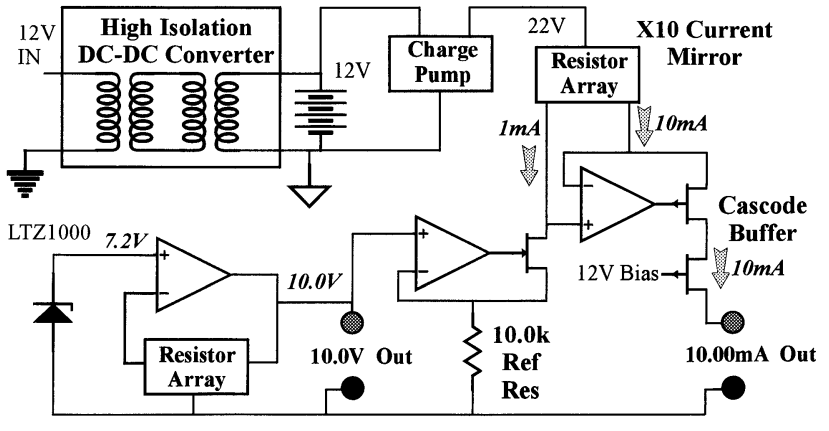

Fig. 4. Block diagram.

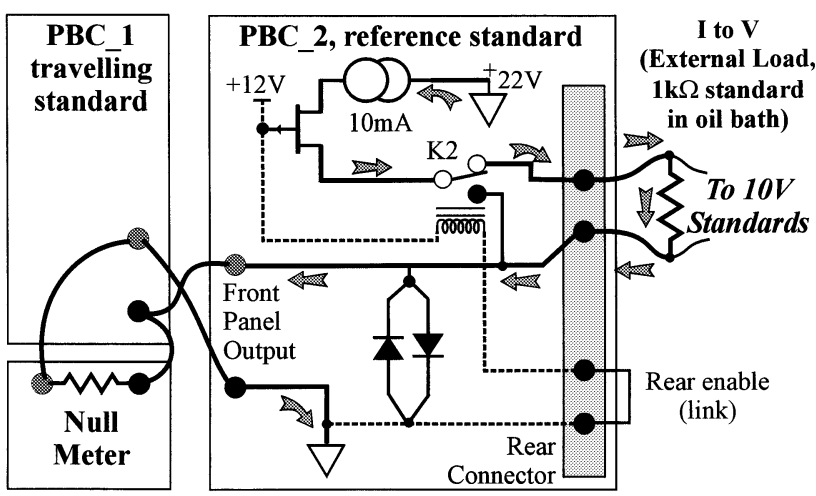

Fig. 5. Current path in voltage to current transfer.

LTZ1000A, at 7.2 V, is defined by "statistical" TaN film resistor arrays [2]. These have been shown to maintain stable ratios to less than one part in $10^{6}$ over time and temperature. The $10 \mathrm{~V}$ is then converted to $1 \mathrm{~mA}$ in a transconductance stage, utilizing a $10-\mathrm{k} \Omega$ resistor constructed from four of Vishay's new "Z-Foil" zero TC resistors. By converting to current at only $1 \mathrm{~mA}$, it was possible to use an optimum $10 \mathrm{k} \Omega$ for this critical part. The 1 $\mathrm{mA}$ is amplified by a factor of 10, defined by further use of TaN film arrays in a 10:1 current mirror referred up to $22 \mathrm{~V}$ to achieve the compliance. This current is made adjustable over \pm 5 parts in $10^{5}$ via a front panel, 10 turn, indicating potentiometer in order to allow the transfer of current between devices to be an exact null, the potentiometer indication being recorded to track drift performance between calibrations.

\section{DESIGN For COMPLIANCE}

The current path for the most stringent dc compliance situation, where reference units actively transfer voltage traceability concurrently to current standards is shown in Fig. 5. In this mode, the reference 1-k $\Omega$ resistor is in series, via PBC_1's rear connector, with the front panel output, which in turn is used in "back-to-back" configuration to calibrate PBC_2.

\section{A. Current Mirror}

In an opamp assisted current mirror, in its simplest form, the output compliance is a function of the loop gain of the controlling opamp. This means that reactive loads can interfere with the performance to the point of becoming unstable. This circuit adds a cascode stage, making it very "stiff" with the opamp being buffered from the output voltage. Furthermore, by using 


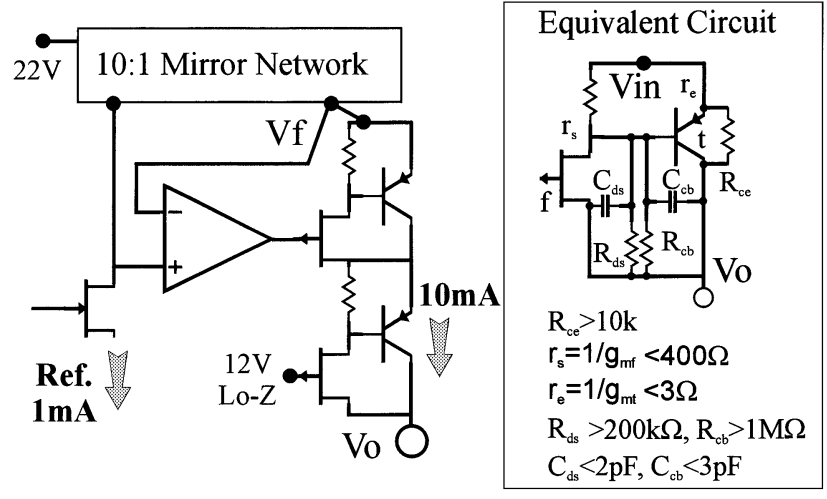

Fig. 6. Double cascode output.

small geometry JFETs and adding bipolar transistors to boost the current handling, an output resistance in excess of $20 \mathrm{G} \Omega$ was achieved, independent of load dynamics. Fig. 6 shows the arrangement along with an equivalent circuit. The reverse attenuation of each stage can then be calculated at dc with the values shown for the significant component characteristics as follows:

$$
\frac{V_{\mathrm{in}}}{V_{o}}=\frac{r_{s}}{R_{d s}}+\frac{r_{s}}{R_{c b}}+\frac{r_{e}}{R_{c e}} \approx \frac{400}{2 E 5}+\frac{400}{1 E 6}+\frac{3}{1 E 4} .
$$

The resultant reverse transfer ratio for the two stages is obtained subsequently by squaring, $V_{f} / V_{o}=\left(V_{\text {in }} / V_{o}\right)^{2}$, which is better than $-100 \mathrm{~dB}$.

Most importantly, this is achieved at the bandwidth of the discrete devices and, since the feedback capacitance is of the order of $5 \mathrm{pF}$, this gives $-3 \mathrm{~dB}$ for each stage at $176 \mathrm{kHz}$. Clearly then, $-100 \mathrm{~dB}$ is maintained to $>100 \mathrm{kHz}$. The output resistance $R_{o}$ is then a function of the reverse gain and the current mirror source resistor, $R_{s}(500 \Omega)$, as follows:

$$
R_{0}=R_{s} \frac{V_{0}}{V_{f}}, \text { which is }>50 \mathrm{M} \Omega .
$$

Multiplying by the opamp loop gain gives $R_{o} \gg 20 \mathrm{G} \Omega$.

\section{B. Thermal Balance}

Naturally, as the compliance voltage changes, so does the local internal power dissipation and this needs correction with the power balance circuit shown in Fig. 7.

\section{DESIGN FOR ISOLATION}

The back-to-back calibration configuration is shown in Fig. 3 and inspection shows that if the power supplies generate common mode current, this will flow through the sense resistor. It, therefore, constitutes an error if it is dc or a disturbance if it is AC. A new dc-dc converter using a patented double-screened transformer construction was chosen, together with a slew-limited low-noise switching controller. This ensures that there will be no difference between use with external power supplied and use with the external supply completely disconnected when under battery power. The double-screened transformer uses two toroidal cores, independently wound, one each for primary and for secondary. Each is fully screened within injection moulded conductive plastic shrouds and coupled

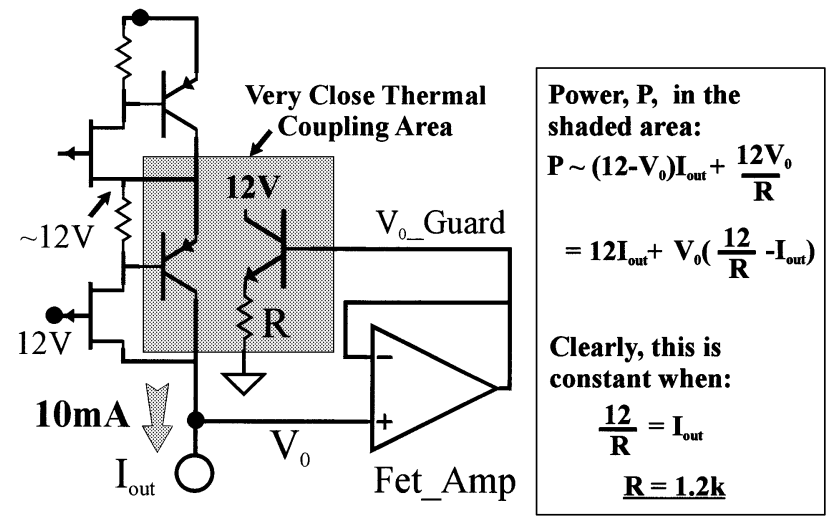

Fig. 7. Thermal balance.

with an external "shorted turn" winding around both cores. The resulting coupling capacitance from primary winding to secondary screen is less than $0.1 \mathrm{pF}$ and in use $<10 \mathrm{nA}$ of pk-pk AC coupling current is achieved compared with at least 1000 times more in most commercial units.

\section{DESIGN FOR PORTABILITY}

The application requires that autonomy is maintained over a working day and that the unit is left on charge whenever not being used for transfers. Consequently, great care was taken to minimize power consumption, which was kept down to about $1.9 \mathrm{~W}$ including trickle charge. The battery chosen for convenience and cost was a "block" of NiMH AA cells with 1200 mAh capacity. However, the trickle charge rate was kept down to $10 \mathrm{~mA}$ so that the internal power dissipation change between line and battery power was minimized. This is essential to minimize possible changes in output voltage or current due to thermal differences. It proved difficult to ascertain the charge state of units with unknown recent history, so a new "Boost" mode was designed, whereby an operator can force a state of fairly rapid charge during which normal use is inhibited. The boost charge takes some $12 \mathrm{~h}$ and would normally be performed overnight. Subsequent to this, an autonomy of $14 \mathrm{~h}$ (with newly available $1600 \mathrm{mAh}$ batteries) can be guaranteed. If the battery discharges completely, it is essential that, when the power is restored, the unit "wakes up" in a state indicating that power has previously failed. This is indicated visually by an LED and signalled to the rear connector so that appropriate action can be taken. For most purposes a calibration reset cycle can be initiated by the user and this is guaranteed, under normal conditions, to return the unit to within five parts in $10^{7}$ of its pre-failure condition, typically to within two parts in $10^{7}$.

\section{CONCLUSION}

All of the design objectives were met and the PBC allows very high performance transfers to be made based on current rather than voltage. It also supplies near error-free current to the current calibrator in the presence of harsh inductive loading and back EMFs. Final accuracy is primarily limited by the reference zener and the internal reference resistor. The design principles could perhaps be the basis for a new class of current standards. 


\section{REFERENCES}

[1] [Online]. Available: http://www.cern.ch, http://lhc.web.cern.ch/lhc/

[2] J. Pickering, "A solid state DC reference system," in NCSL Conf. Dig., 1995 , p. 369

[3] G. Fernqvist, "The measurement challenge of the LHC project," IEEE Trans. Instrum. Meas., vol. 48, pp. 462-466, Apr. 1999.

[4] G. Fernqvist, B. Halvarsson, J. Pett, and J. Pickering, "A novel current calibration system up to $20 \mathrm{kA}$," IEEE Trans. Instrum. Meas., vol. 52, no. Apr., 2003.

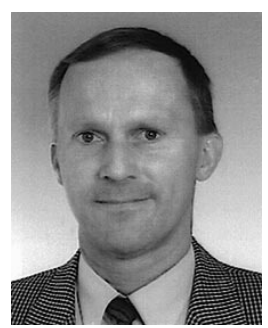

Gunnar Fernqvist (M'95) was born in Stockholm, Sweden, in 1944. He received the M.Sc. degree in electronics from the Royal Institute of Technology, Stockholm, in 1969.

In 1972, he joined the European Organization for Nuclear Research (CERN) as Project Engineer in power converter remote control with special interest in high precision $\mathrm{A} / \mathrm{D}$ and $\mathrm{D} / \mathrm{A}$ conversion. Between 1974 and 1982, he was responsible for the operation of the Intersecting Storage Rings (ISR) power converters and the beginnings of the CERN Standards Laboratory. From 1983 to 1992, he was responsible for the design and construction of the high voltage power networks for the LEP accelerator. From 1993 to 2001, he was the head of the SPS/LEP power converter group and also directed the activities of the Standards laboratory. He is now responsible for the high precision aspects of the SPS and LHC power converters.

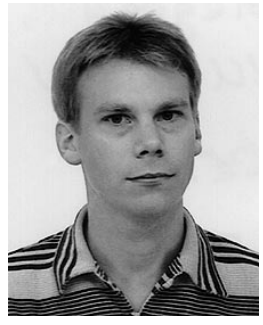

Gregory Hudson was born in Oxfordshire, U.K., in 1967. He received the B.Sc. degree from Open University, Milton Keynes, U.K. in 1998.

In 1984, he joined the U.K. Atomic Energy Authority, Harwell, where he did his technical studies and electronics training. In 1989, he joined the Joint European Torus (JET) project, U.K., where he worked as a Technician in both the Remote Handling and Power Supplies Groups. In 1995, he joined the European Organization for Nuclear Research (CERN), Geneva, Switzerland, as a Beam Line Technician in the experimental areas. In 1998, he moved to the Standards Laboratory, where he now works as a Technical Engineer.

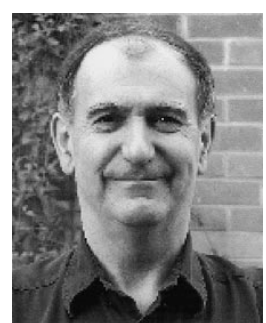

John Pickering (M'77) received the B.Sc. degree (hons) in electrical engineering from Imperial College, London, U.K., in 1967.

He pursued a chosen career in electronic measurement and instrumentation at Hewlett Packard (now Agilent) in Edinburgh, U.K., and Dana Laboratories in Irvine, CA, and Luton, U.K. In 1971, he cofounded Datron Electronics Ltd., Norwich, U.K. (Now Fluke Precision Measurement Ltd.), modeling it on Hewlett Packard's management methods of the time. Generally. he made direct circuit and system level contributions to nearly all of the Datron products; indeed, in the earlier stages of the company's development, he was the main product Architect and Circuit Designer. He has 35 years of experience in electronic metrology and currently runs Metron Designs Ltd., a contract design company specializing in designs for precision dc and LF measurement such as long scale A-Ds and D-As, ultralow noise power supplies, and precision reference sources.

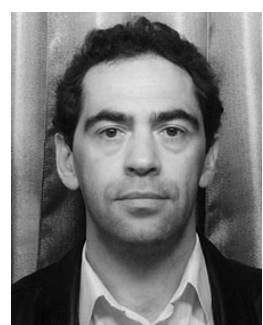

Francis Power was born in Liverpool, U.K., in 1956. He received the B.Sc. degree in electrical and electronics engineering from Leeds University, Leeds, U.K., in 1978.

In 1978, he joined the DVM group of Solarton Instruments, Farnborough, U.K., as a Design Engineer. From 1978 to 1990, he designed many high precision DVMs, and was ultimately responsible for all DVM R\&D activities. In 1991, he joined Orbisphere Laboratories, Geneva, Switzerland, were he was responsible for ATE test systems, the computing system, and installing an ISO9001 quality system. In 1998, he joined the Standards Laboratory at the European Organization for Nuclear Research (CERN), Geneva, Geneva, and is currently responsible for automating the various test beds for testing high-precision direct current current transducers (DCCTs) and managing the DCCT contracts for the LHC project. 